\title{
Use of probiotics in pediatric patients with autism spectrum disorder: a systematic review
}

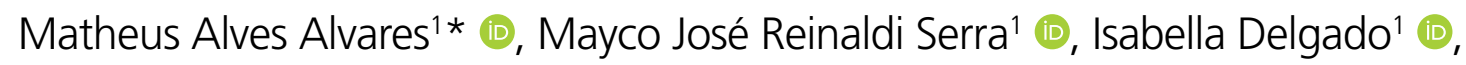 \\ Joyce Canato de Carvalho' ${ }^{10}$, Thaís Campanhã Cury Sotine ${ }^{1}$ (D), Youssef Adef Ali' ${ }^{10}$, \\ Marcella Rocha Machado de Oliveira² (1), Vera Esteves Vagnozzi Rullo
}

\section{INTRODUCTION}

Autism spectrum disorder (ASD) is a neurodevelopmental disorder, characterized by persistent impairment in communication and social interaction, in addition to repetitive and stereotyped behaviors ${ }^{1}$. Approximately $50 \%$ of patients with ASD also have gastrointestinal (GI) symptoms, mainly constipation, abdominal pain, diarrhea, flatulence, and vomiting ${ }^{2}$.

According to the study by Karimi ${ }^{3}$, the etiology of ASD is multifactorial, involving environmental and genetic factors. Recent studies ${ }^{4-6}$ indicate dysbiosis of the intestinal microbiota as an important factor in its development and in other neuropsychiatric diseases, such as depression and Parkinson's disease ${ }^{7}$.

A review of the literature showed that children with ASD have a greater abundance of Bacteroides, Parabacteroides, Clostridium, Faecalibacterium, and Phascolarctobacterium colonizing them, differing from the pattern of colonization of neurotypical children who generally have a higher prevalence of Coprococcus and Bifidobacterium ${ }^{8}$.

Defined by the World Health Organization (WHO) as "Live microorganisms that, when administered in adequate quantities, confer a health benefit," probiotics have gained prominence after studies suggest that they could be a useful therapeutic tool to alter brain function by its activity in restoring the healthy balance of the intestinal microbiota and modulating the levels of neurotransmitters ${ }^{10-12}$.

As an example, the hypothalamic-pituitary-adrenal (HPA) stress response, which controls emotion and mood, can be attenuated by certain probiotic microorganisms, thus decreasing the levels of corticosteroids. In the immune system, some probiotics can restrict the production of pro-inflammatory cytokines and can alter metabolites such as tryptophan and short-chain fatty acids, which help to regulate the cellular immune response ${ }^{13}$.

This study aimed to provide an updated review in order to clarify the effect of the use of probiotics, when compared to placebo, in the behavioral aspect and in the gastrointestinal tract (GIT) of pediatric patients with ASD.

\section{METHODS}

This systematic review of randomized controlled trials (RCTs) evaluated the effects of probiotics on the behavioral issue and on the GIT of pediatric patients with ASD. The articles were selected according to the recommendations of the Preferred Reporting Items for Systematic Reviews and Meta-analysis (The PRISMA Statement $)^{14}$, responsible for coordinating the process of making meta-analyses and systematic reviews.

For the selection, a systematic search of the literature was carried out in the databases Medline (via PubMed), LILACS (via Virtual Health Library), and SciELO. The following search terms were used in the Medline databases: (probiotics) AND (autism OR ASD); LILACS: (“"Autistic Spectrum Disorder”) AND ("probiotics”)); SciELO: (probiotics) AND (autism OR ASD). The surveys were carried out between February and March 2021, without language restrictions.

The inclusion criteria for this review included only the placebo-controlled RCTs, the studies carried out with the pediatric population diagnosed with ASD and intervention with probiotics. The exclusion criteria were observational studies, studies in the non-pediatric population, literature reviews, duplicate studies, interventions without the use of probiotics, and studies with patients with diseases associated with ASD. After a

${ }^{1}$ Centro Universitário Lusíada - Santos (SP), Brazil.

IIrmandade Santa Casa de Misericórdia de São Paulo - São Paulo (SP), Brazil.

*Corresponding author: matheusalvares.ep@gmail.com

Conflicts of interest: the authors declare there are no conflicts of interest. Funding: none.

Received on July 04, 2021. Accepted on August 10, 2021. 
systematic review of the literature using the databases mentioned above, the articles that met the inclusion criteria were selected. A total of 215 articles were found as follows: 187 via Medline, 27 via LILACS, and 1 via SciELO. After the methodological screening, two studies were eligible for this systematic review. Figure 1 describes the steps for selecting the articles.

\section{RESULTS}

The two studies selected for this review are RCTs, containing a control group receiving placebo and an intervention group receiving probiotics, in a pediatric population diagnosed with ASD, totalizing 134 patients.

The studies were evaluated individually for methodological quality (risk of bias) following Cochrane's Risk of Bias Tool $(\text { RoB- } 2)^{15}$, which is more detailed in Table 1.

In 2019, to study the impacts of Lactobacillus plantarum PS128 on the brain-intestine axis, Liu et al. ${ }^{16}$ underwent a four-week double-blind RCT with 71 male patients between 7 and 15 years (36 controls and 35 interventions) diagnosed with ASD. Candidates who had taken antibiotics, yogurts, or probiotic products two weeks prior to registration were excluded. Participants were allowed to continue their regular medications, treatment, and therapies, with the exception of antibiotics, and were asked to refrain from consuming yogurt or probiotic products during the study period. The results were evaluated from the questionnaires, such as Autism Behavior

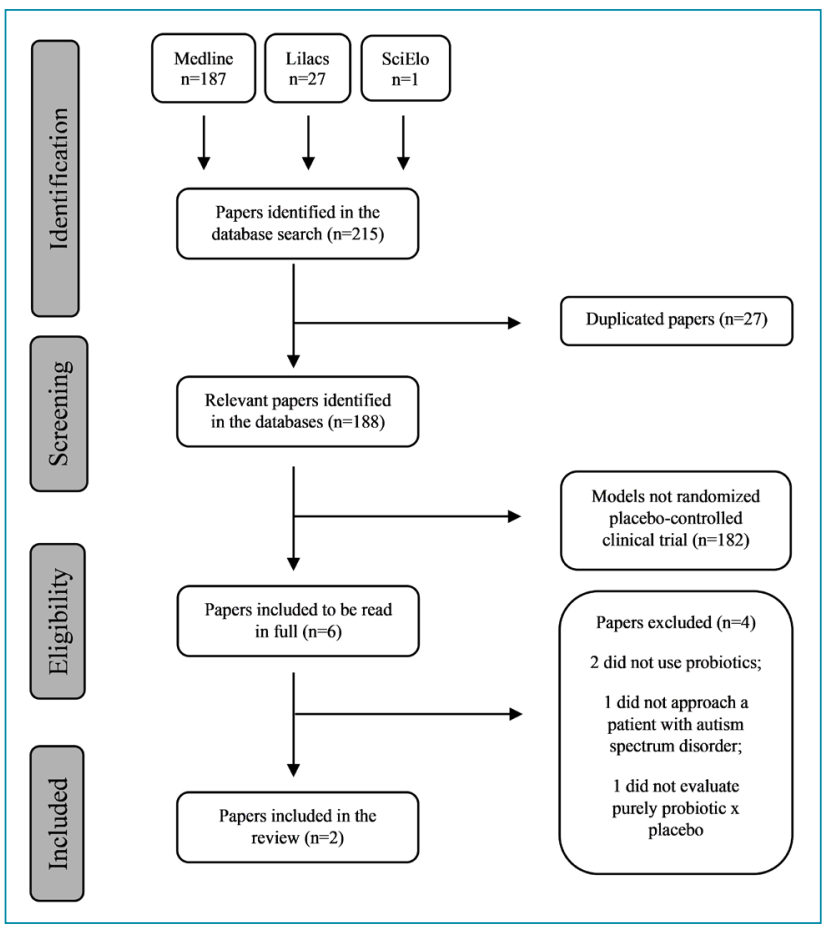

Figure 1. Flowchart of studies included in the analysis.
Checklist-Taiwan (ABC-T); Social Responsiveness Scale (SRS); Child Behavior Checklist (CBCL); Multimodal Treatment Study for Attention Deficit Hyperactivity Disorder (ADHD), version of Swanson, Nolan, and Pelham, Version IV, adapted for Brazil (SNAP-IV). After 4 weeks, there was a statistical significance in the reduction of the total scores of SRS $(\mathrm{p}=0.04)$ and SNAP-IV $(\mathrm{p}=0.02)$ of the group that received treatment with probiotics, a fact not observed in the placebo group. In addition, in the treated group, exploratory analyses revealed improvement in anxiety and rule-breaking behaviors $(\mathrm{p}=0.02)$ in CBCL and improvement in relation to body and object use $(\mathrm{p}=0.04)$ in the ABC-T. In SNAP-IV, there was an improvement in hyperactivity and impulsivity $(\mathrm{p}=0.04)$. No adverse events, GI intolerance, or allergic response were reported by parents or participants.

The study by Santocchi et al. ${ }^{17}$ aimed to evaluate the use of the probiotic mixture Vivomixx ${ }^{\circledR}$ in pediatric patients diagnosed with ASD who have GI symptoms and non-GI (NGI) symptoms. The primary outcome was to evaluate the improvement in the level of severity of ASD symptomatology through the Autism Diagnostic Observation Schedule Calibrated Severity Score (ADOS-CSS), and the secondary outcome was to evaluate the improvement of GI symptoms, assessed by the Gastrointestinal Severity Index (GSI). The exclusion criteria were neurological syndromes or focal neurological signs, epilepsy, history of neonatal asphyxia, severe prematurity, and other perinatal lesions; significant sensory deficiency (e.g., blindness and deafness); and diagnosis of nonfunctional GI disorder or celiac disease and special diets already underway. A double-blind and parallel RCT was conducted in 63 children aged 18-72 months, the control group composed of eight GI and 24 NGI children, and the intervention group consisted of nine GI and 22 NGI children. Therapy was applied at a dose

Table 1. Cochrane's Risk of Bias Tool ${ }^{15}$.

\begin{tabular}{l|c|c} 
Criteria & $\begin{array}{c}\text { Liu } \\
\text { et al. }\end{array}$ & $\begin{array}{c}\text { Santocchi } \\
\text { et al. }{ }^{17}\end{array}$ \\
\hline 1. Clear objective & A & A \\
\hline 2.Suitable sample size & B & B \\
\hline $\begin{array}{l}\text { 3.Sample identification } \\
\text { and evaluation }\end{array}$ & A & A \\
\hline 4. Comparability & B & A \\
\hline 5. Blinding of participants & A & A \\
\hline 6. Other bias & C & B \\
\hline 7. Proper statistical analysis & B & B \\
\hline Total & C & C \\
\hline
\end{tabular}

A: low risk of bias; B: intermediate risk of bias; C: high risk of bias. 
of two sachets/day in the first month and one sachet/day in the following 5 months (with the placebo mixture being identical to the intervention). After 6 months of treatment, the ADOS-CSS did not obtain a statistically significant difference. However, in an exploratory analysis, the NGI group treated with probiotics showed a significant reduction $(\mathrm{p}=0.026)$ in the total ADOS-CSS (which decreased from 6.72-5.91 in the probiotic group and increased from $6.96-7.17$ in the placebo group). In addition, GI patients presented a statistically significant reduction in GI symptoms (6-GSI [ $\mathrm{p}=0.009])$, mainly in smell in feces and flatulence $(\mathrm{p} \leq 0.001)$, including a higher proportion of children with the normalization of sensory profile scores in the Multisensory Processing subscale ( $\mathrm{p}=0.013)$.

Table 1 shows the statistical results and Table 2 shows the results found in the study.

\section{DISCUSSION}

Probiotics have recently been used in several clinical trials as an additional treatment combined with conventional therapy in patients with ASD. This is due to the fact of the difference in the colonization of its microbiota when compared to that of neurotypical patients ${ }^{8}$. Children with ASD and GI symptoms have shown high levels of intestinal inflammation associated with dysbiosis ${ }^{5,6,18}$ The probiotic approach should act as a tool to restore the healthy microbiota, in addition to reducing intestinal permeability and making negative regulation of inflammatory cytokines ${ }^{19}$.

Regarding the analyzed studies, it is possible to observe heterogeneity regarding the use of probiotics (i.e., one study used a mixture and the other an isolated strain), and the dose used was variable, as well as the follow-up time. The studies were conducted in two different countries, which may lead to a broader evaluation, being a positive point observed.

The limitations of the studies include the reduced number of articles analyzed and the follow-up time, which limits the evaluation until the short term. The levels of markers of intestinal inflammation, as well as the evaluation of the intestinal microbiota made before and after the intervention with probiotic, did not present significant values in one study and was not performed in another, so there was no direct demonstration of its effects, focusing only on questionnaires performed.

The studies included in this article ${ }^{16,17}$ demonstrated empirical improvements reported by parents and patients; however, there was less statistical significance in the reduction of total scores in the main questionnaires evaluated (i.e., CGI-I, ABCT, ADOS-CSS, PedQL, and PRAS-ASD), with the exception

Table 2. Behavioral assessment by questionnaires and evaluation of symptom severity in patients with gastrointestinal symptoms.

\begin{tabular}{l|c|c|c|c|c|c}
\hline Study & Parameter & PR T & PR T $_{1}$ & PL T & PL T $_{1}$ & p-valuet \\
\cline { 2 - 7 } & ABC-T & $15.81(8.39)$ & $14.67(8.97)$ & $17(9.31)$ & $16.21(10.11)$ & 0.53 \\
\cline { 2 - 7 } Liu et al. & SRS & $138.87(24.19)$ & $132.77(22.99)$ & $135.88(26.04)$ & $135.79(25.79)$ & 0.63 \\
\cline { 2 - 7 } & CBCL & $49.63(25.4)$ & $44.34(23.25)$ & $50.60(25.91)$ & $49.20(24.46)$ & 0.53 \\
\cline { 2 - 7 } & SNAP-IV & $34.03(14.61)$ & $31.87(14.26)$ & $34.48(13.39)$ & $33.16(15.58)$ & 0.73 \\
\hline \multirow{4}{*}{$\begin{array}{l}\text { Santocchi } \\
\text { et al. }{ }^{17}\end{array}$} & ADOS-CSS & $6.84(1.39)$ & $6.19(1.56)$ & $6.97(1.91)$ & $7.00(1.80)$ & NS \\
\cline { 2 - 7 } & SCQ & $12.83(6.68)$ & $11.97(6.71)$ & $16.06(5.54)$ & $13.90(6.19)$ & NS \\
\cline { 2 - 7 } & RBS-R & $18.32(13.17)$ & $14.37(8.01)$ & $22.31(15.47)$ & $19.13(12.10)$ & NS \\
\cline { 2 - 7 } & DQ & $65.91(18.06)$ & $69.27(20.09)$ & $62.29(20.12)$ & $61.14(20.13)$ & NS \\
\cline { 2 - 7 } & VABS I & $63.87(22.12)$ & $67.39(22.29)$ & $57.00(16.74)$ & $59.72(16.38)$ & NS \\
\cline { 2 - 7 } & CBCL & $60.94(9.94)$ & $57.80(7.92)$ & $62.84(10.97)$ & $57.30(9.05)$ & NS \\
\hline \multirow{4}{*}{$\begin{array}{l}\text { Santocchi } \\
\text { et al. }{ }^{17}\end{array}$} & PSI & $70.03(29.63)$ & $66.62(31.15)$ & $74.76(24.98)$ & $61.03(32.58)$ & NS \\
\cline { 2 - 7 } & GSI, Smell of feces & $1.88(0.33)$ & $0.56(0.88)$ & $0.25(0.71)$ & $0.14(0.38)$ & $<0.001$ \\
\cline { 2 - 7 } & GSI, Flatulence & $0.56(0.88)$ & $0.33(0.50)$ & $0.43(0.79)$ & $0.86(0.99)$ & 0.0187 \\
\cline { 2 - 7 } & GSI, Total & $7.22(1.99)$ & $2.89(2.31)$ & $5.75(1.03)$ & $3.43(1.81)$ & 0.0416 \\
\hline
\end{tabular}

t: p-value among the groups at the end of the intervention. Results expressed in standard deviation (SD). PR: probiotic; PL: placebo; NS: not significant; $\mathrm{T}_{0}$ : zero time; $\mathrm{T}_{1}$ : final time; ABC-T: autism behavior checklist-Taiwan; SRS: Social responsiveness scale; CBCL: Child behavior checklist; PSI: Parental stress index; SNAP-IV: Study version of the Swanson, Nolan, and Pelham, Version IV Scale; ADOS-CSS: Autism diagnostic observation schedule calibrated severity score; SCQ: Social communication questionnaire; RBS-R: Repetitive behavior scale-revised; VABS-II: Vineland adaptive behavior scales-second edition; GSI: Gastrointestinal severity index. 
of SNAP-IV, which obtained a significant reduction $(\mathrm{p}=0.02)$ in the total score ${ }^{17}$, especially in the group of younger patients (7-12 years) $(p=0.004)^{16}$. They also showed better responses in the symptoms of inattention, hyperactivity/impulsivity, opposition/challenge, and rule-breaking behaviors ${ }^{17}$, suggesting that younger patients may respond better to probiotic therapy in terms of behavioral aspects.

Santocchi et al. ${ }^{17}$ divided patients with and without GI symptoms, obtaining different results among them, because only the NGI group, treated with probiotics, showed a significant reduction in ADOS-CSS (Total and AffectiveSocial scores), while patients in the GI group who received probiotics obtained improvement only in GI symptoms, with emphasis on reducing flatulence and smell in feces. These results corroborate other articles ${ }^{20-22}$ that indicate the positive effect on the use of probiotics in patients with ASD, to improve GI symptoms such as constipation, abdominal pain, reduction of diarrhea, and improvement of stool consistency. Santocchi et al. ${ }^{17}$ also indicated that the disparity between the results obtained by them can be explained by the heterogeneity in the composition of the microbiota of the participants, causing there to be potentially different effects on different targets.
In view of these results, the approach with probiotics showed low efficacy in improving behavioral symptoms, with some favorable outcomes ${ }^{18}$ in patients with GI complaints, which could justify its use in complementary therapies. However, larger studies, with the laboratory microbiota analysis for better direction, should be conducted to attest or not to the efficacy of probiotic therapy in pediatric patients with ASD.

\section{AUTHORS" CONTRIBUTIONS}

MAA: Conceptualization, Formal analysis, Methodology, Project administration, Supervision, Writing - original draft, Writing - review \& editing. MJRS: Formal analysis, Supervision, Writing - original draft, Writing - review \& editing. ID: Investigation, Methodology, Project administration, Writing - original draft. JCC: Investigation, Methodology, Project administration, Writing - original draft. TCCS: Investigation, Methodology, Project administration, Writingoriginal draft. YAA: Investigation, Methodology, Project Administration, Writing - original draft. MRMO: Validation, Resources, Writing - original draft, Writing - review \& editing. VEVR: Supervision, Validation, Writing- review \& editing.

\section{REFERENCES}

1. American Psychiatric Association. (2013). Neurodevelopmental disorders. Autism spectrum disorder. In: American Psychiatric Association. Diagnostic and statistical manual of mental disorders. 5th ed. Arlington: American Psychiatric Association; 2013.

2. Buie T, Campbell DB, Fuchs GJ 3rd, Furuta GT, Levy J, Vandewater $J$, et al. Evaluation, diagnosis, and treatment of gastrointestinal disorders in individuals with ASDs: a consensus report. Pediatrics. 2010;125(Suppl 1):S1-18. https://doi.org/10.1542/ peds.2009-1878C

3. Karimi P, Kamali E, Mousavi SM, Karahmadi M. Environmental factors influencing the risk of autism. J Res Med Sci. 2017;22:27. https://doi.org/10.4103/1735-1995.200272

4. McElhanon BO, McCracken C, Karpen S, Sharp WG. Gastrointestinal symptoms in autism spectrum disorder: a meta-analysis. Pediatrics. 2014;133(5):872-83. https://doi. org/10.1542/peds.2013-3995

5. Finegold SM, Molitoris D, Song Y, Liu C, Vaisanen ML, Bolte $\mathrm{E}$, et al. Gastrointestinal microflora studies in late-onset autism. Clin Infect Dis. 2002;35(Suppl 1):S6-16. https://doi. org/10.1086/341914

6. Mangiola F, laniro G, Franceschi F, Fagiuoli S, Gasbarrini G, Gasbarrini A. Gut microbiota in autism and mood disorders. World J Gastroenterol. 2016;22(1):361-8. https://doi. org/10.3748/wjg.v22.i1.361
7. Ansari F, Pourjafar $H$, Tabrizi A, Homayouni A. The effects of probiotics and prebiotics on mental disorders: a review on depression, anxiety, Alzheimer, and autism spectrum disorders. Curr Pharm Biotechnol. 2020;21(7):555-65. https://doi.org/1 $0.2174 / 1389201021666200107113812$

8. Iglesias-Vázquez L, Van Ginkel Riba G, Arija V, Canals J. composition of gut microbiota in children with autism spectrum disorder: a systematic review and meta-analysis. Nutrients. 2020;12(3):792. https://doi.org/10.3390/nu12030792

9. The Food and Agriculture Organization. Probiotics in food. Health and nutritional properties and guidelines for evaluation. Rome: The Food and Agriculture Organization; 2006.

10. Liu J, Wan GB, Huang MS, Agyapong G, Zou TL, Zhang XY, et al. Probiotic therapy for treating behavioral and gastrointestinal symptoms in autism spectrum disorder: a systematic review of clinical trials. Curr Med Sci. 2019;39(2):173-84. https:// doi.org/10.1007/s11596-019-2016-4

11. Martínez-González AE, Andreo-Martínez P. Prebiotics, probiotics and fecal microbiota transplantation in autism: a systematic review. Rev Psiquiatr Salud Ment. 2020;13(3):150-64. https:// doi.org/10.1016/j.rpsm.2020.06.002

12. Ng QX, Loke W, Venkatanarayanan N, Lim DY, Soh AYS, Yeo WS. A systematic review of the role of prebiotics and probiotics in autism spectrum disorders. Medicina. 2019;55(5):129. https://doi.org/10.3390/medicina55050129 
13. Wang $\mathrm{H}$, Lee IS, Braun $\mathrm{C}$, Enck P. Effect of probiotics on central nervous system functions in animals and humans: a systematic review. J Neurogastroenterol Motil. 2016;22(4):589-605. https://doi.org/10.5056/jnm16018

14. Moher D, Liberati A, Tetzlaff J, Altman DG; PRISMA Group. Preferred reporting items for systematic reviews and meta-analyses: the PRISMA statement. PLoS Med. 2009;6(7):e1000097. https://doi.org/10.1371/journal. pmed.1000097

15. Sterne JAC, Savović J, Page MJ, Elbers RG, Blencowe NS, Boutron I, et al. RoB 2: a revised tool for assessing risk of bias in randomised trials. BMJ. 2019;366:|4898. https://doi. org/10.1136/bmj.14898

16. Liu YW, Liong MT, Chung YE, Huang HY, Peng WS, Cheng YF, et al. Effects of lactobacillus plantarum ps 128 on children with autism spectrum disorder in Taiwan: a randomized, doubleblind, placebo-controlled trial. Nutrients. 2019;11(4):820. https://doi.org/10.3390/nu11040820

17. Santocchi E, Guiducci L, Prosperi M, Calderoni S, Gaggini $M$, Apicella $F$, et al. Effects of probiotic supplementation on gastrointestinal, sensory and core symptoms in autism spectrum disorders: a randomized controlled trial. Front Psychiatry. 2020;11:550593. https://doi.org/10.3389/fpsyt.2020.550593
18. Arnold LE, Luna RA, Williams K, Chan J, Parker RA, Wu Q, et al. Probiotics for gastrointestinal symptoms and quality of life in autism: a placebo-controlled pilot trial. J Child Adolesc Psychopharmacol. 2019;29(9):659-69. https://doi.org/10.1089/ cap.2018.0156

19. Parracho HM, Bingham MO, Gibson GR, McCartney AL. Differences between the gut microflora of children with autistic spectrum disorders and that of healthy children. J Med Microbiol. 2005;54(Pt 10):987-91. https://doi.org/10.1099/jmm.0.46101-0

20. Li Q, Zhou JM. The microbiota-gut-brain axis and its potential therapeutic role in autism spectrum disorder. Neuroscience. 2016;324:131-9. https://doi.org/10.1016/j. neuroscience.2016.03.013

21. West R, Roberts E, Sichel LS, Sichel J. Improvements in gastrointestinal symptoms among children with autism spectrum disorder receiving the Delpro ${ }^{\circledR}$ probiotic and immunomodulator formulation. J Probiotics Health. 2013,1(2):1-6. https://doi. org/10.4172/2329-8901.1000102

22. Shaaban SY, El-Gendy YG, Mehanna NS, El-Senousy WM, El-Feki HSA, Saad K, et al. The role of probiotics in children with autism spectrum disorder: a prospective, open-label study. Nutr Neurosci. 2018;21(9):676-81. https://doi.org/ 10.1080/1028415X.2017.1347746 